\title{
Unraveling the Early-Stage Ordering of Krypton Solid Bubbles in Molybdenum: A Multi- Modal Study
}

Ericmoore Jossou ${ }^{\mathrm{a}}$, Anton Schneider ${ }^{\mathrm{b}}$, Cheng Sun ${ }^{\mathrm{c}}$, Yongfeng Zhang ${ }^{\mathrm{b}}$, Shirish Chodankar ${ }^{\mathrm{d}}$, Dmytro Nykypanchuk ${ }^{\mathrm{e}}$, Jian Gan ${ }^{\mathrm{c}}$, Lynne Ecker ${ }^{\mathrm{a}}$ Simerjeet K. Gill ${ }^{\mathrm{a}}$

${ }^{a}$ Nuclear Science and Technology Department, Brookhaven National Laboratory, Upton, NY 11973, USA;

${ }^{b}$ Engineering Physics Department, University of Wisconsin, Madison, WI 53706, USA;

${ }^{c}$ Idaho National Laboratory, Idaho Falls ID 83415-6000, USA;

${ }^{d}$ Photon Sciences, Brookhaven National Laboratory, Upton, NY 11973, USA;

${ }^{e}$ Brookhaven National Laboratory Center for Functional Nanomaterials, Upton, NY, 11973, USA. Corresponding authors: ejossou@bnl.gov, gills@bnl.gov

\section{Computational details}

The atomic kinetic Monte Carlo (AKMC) method and the description of He are similar to the work of Sun et al. ${ }^{1}$. Following Deo et al. ${ }^{2}$, a He atom can either occupy an octahedral site as an interstitial or a bcc site as a substitution. Similar to the Mo/V/SIA system ${ }^{3}$, pairwise bonds within the second nearest-neighbor cutoff were used to describe the interaction between He atoms and that between a He atom and a vacancy or a Mo atom. The interaction between a He atom and an SIA was not considered in the current model. The bond energies were fitted to the energetics of $\mathrm{He}$ and $\mathrm{He} / \mathrm{V}$ clusters in bcc Mo from density-function theory calculations. The data used in the fitting include the formation energies of He interstitial $(5.28 \mathrm{eV})$, He substitutional $(4.64 \mathrm{eV}), \mathrm{HeV}_{2}$ cluster (6.81 $\mathrm{eV}), \mathrm{He}_{2} \mathrm{~V}$ cluster $(7.25 \mathrm{eV})$, and $\mathrm{He}_{2} \mathrm{~V}_{2}$ cluster $(8.15 \mathrm{eV})$ in bcc $\mathrm{Mo}^{4}$, and the binding energy between two He interstitials $(0.97 \mathrm{eV})^{5}$. It is further assumed that a pure bcc/octahedral He phase has zero cohesive energy. All pairwise bond energies are summarized in the Supplemental Materials of Ref. ${ }^{1}$. For all simulations, the simulated cell is periodic with a dimension of $80 \times 80$ $\times 80 a_{0}^{3}$, with $a_{0}$ being the bcc Mo lattice parameter. More details regarding the modeling method are described in Ref. ${ }^{1}$ and its Supplemental Materials.

In order to capture the differences between $\mathrm{He}$ gas bubble superlattice (GBS) and $\mathrm{Kr}$ solid bubble superlattice (SBS) formation, a parametric study was performed by identifying the key differences between $\mathrm{Kr}$ and He properties (from Density Functional Theory (DFT) results), and by modifying the parameters of the model accordingly in order to evaluate their impact on the simulation results. We know from an earlier $a b$ initio study that the $\mathrm{Kr}$-vacancy binding energy is $8.64 \mathrm{eV}$, which is much higher than the $3.65 \mathrm{eV} \mathrm{He}$-vacancy binding energy. Also, DFT results show that $2 \mathrm{He}$ atoms to a vacancy is required to prevent its recombination with a SIA, while the same effect is obtained with only $1 \mathrm{Kr}$ atom 6 . The AKMC simulations were performed at temperature of $573 \mathrm{~K}$ with a dose rate of $1 \mathrm{dpa} / \mathrm{s}$ and a gas production ratio of $1000 \mathrm{appm} / \mathrm{dpa}$. These combinations of parameters were chosen in order to form a highly ordered He GBS.

The ordering of a SBS is estimated by extracting the center of mass position of each cluster follow by calculating the structural factor $\mathrm{S}(\mathrm{k})$ over a sampling of $\mathrm{k}$ vectors:

$$
S(k)=\left[\left(\sum_{i}^{N} \cos \left(k \cdot r_{i}\right)\right)^{2}+\left(\sum_{i}^{N} \sin \left(k \cdot r_{i}\right)\right)^{2}\right] / N^{2}
$$


where $N$ is the number of clusters and $r_{i}$ is the position vector of the center of mass of cluster $i$. The maximum value of $\mathrm{S}(\mathrm{k})$ is selected as the "maximum ordering" of the superlattice, and the corresponding wavelength provides the superlattice constant. The critical dose in this work is defined as the dose at which the second derivative of the number of vacancies as a function of dose changes sign (the inflection points of the number of vacancies as a function of the dose).

The ordering of a SBS is estimated by extracting the center of mass of each cluster and by calculating the maximum value of the structural factor $S(k)$.

\section{Results}
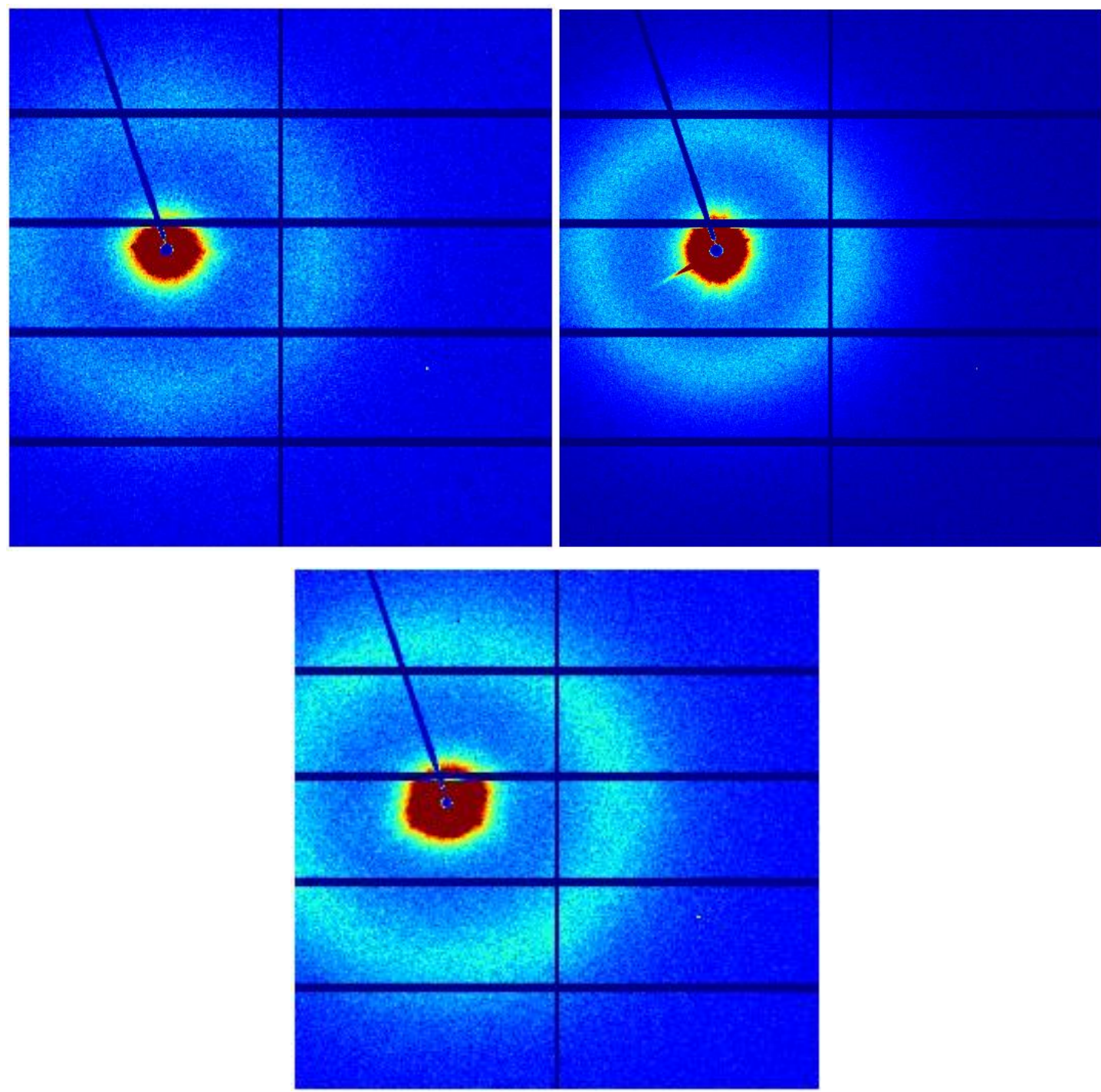

Figure S1. An enlarged 2D diffraction patterns of $2 \mathrm{c}, \mathrm{f}$ and $\mathrm{g}$ respectively to further show the degree of weak ordering as reported in Figure 2. 




Figure S2. The 2D datasets for fluence of $4.5 \times 10^{16} \mathrm{Kr} / \mathrm{cm}^{2}$ at $400{ }^{\circ} \mathrm{C}$ demonstrate the presence of orientation anisotropy in the sample. 

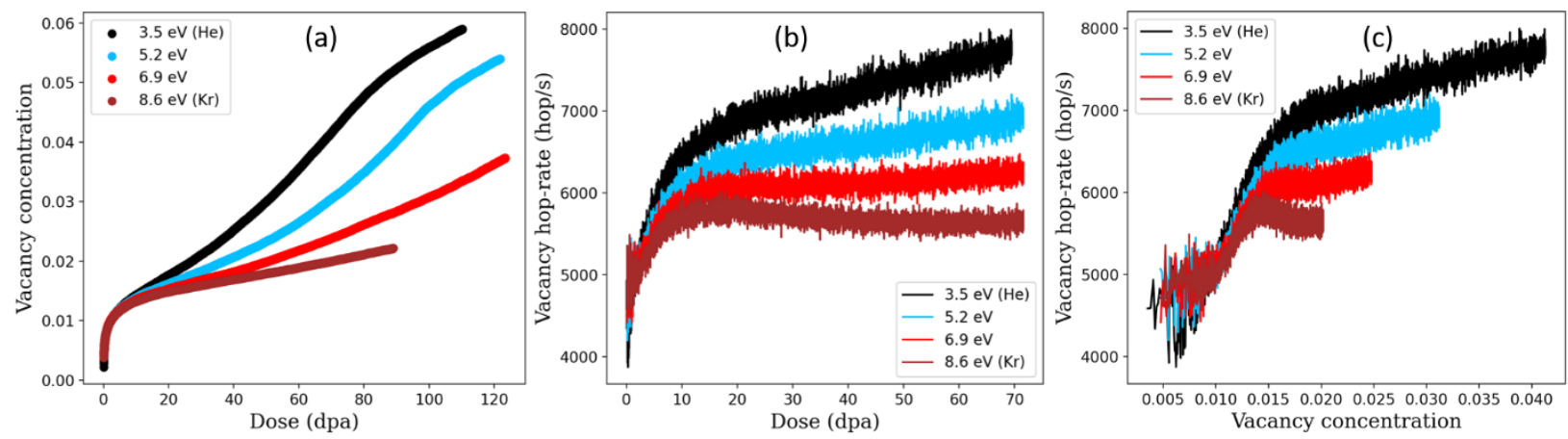

Figure S3. (a) Vacancy concentration as a function of irradiation dose, (b) Vacancy mobility (hop-rate) as a function of irradiation dose, (c) Vacancy mobility as a function of vacancy concentration.

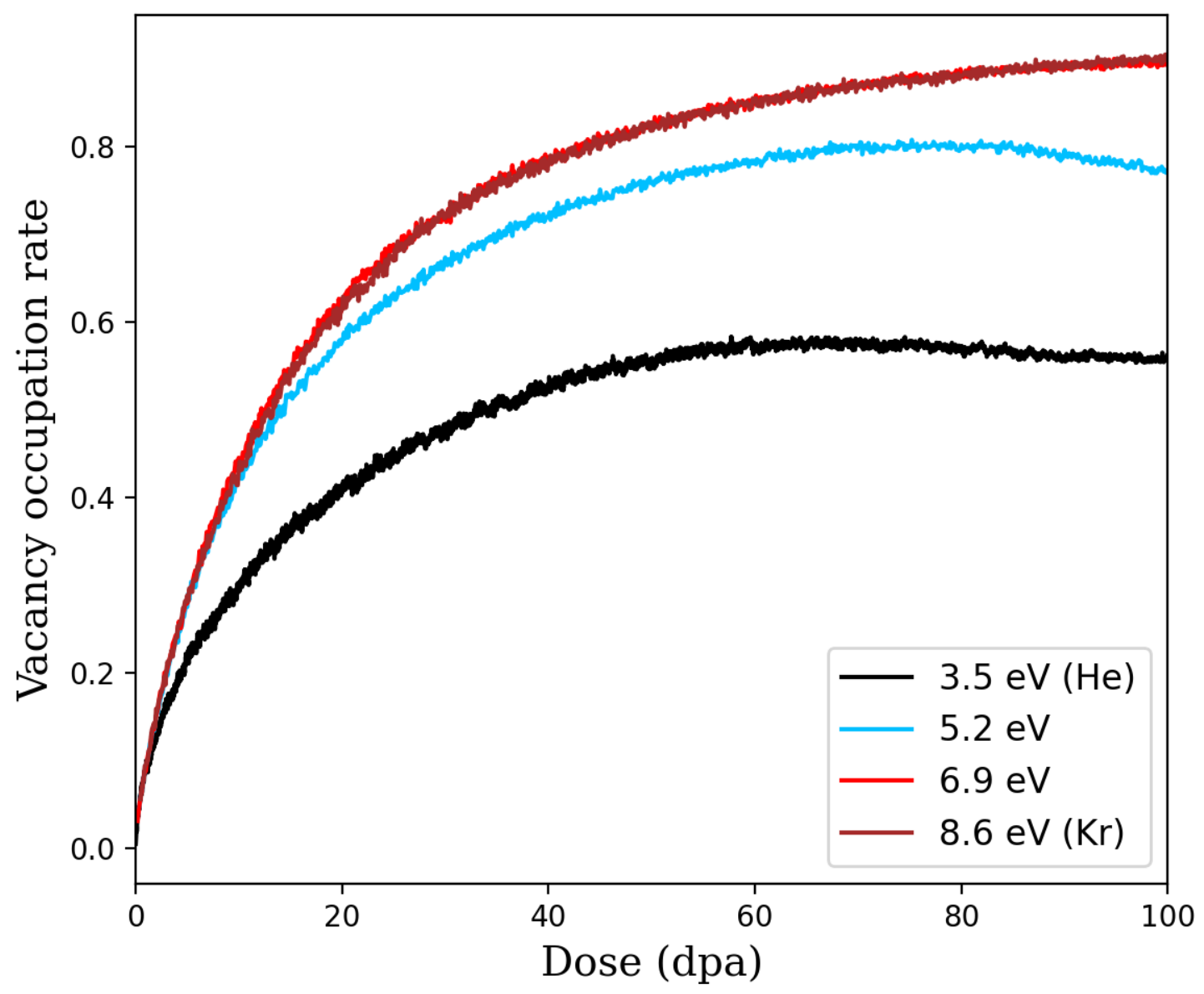

Figure S4. Proportion of vacancies that are occupied by a gas atom, for various gas-vacancy binding energy values. The recombination rate of Frenkel pairs for that factor is not reliable as the SBS starts its formation while the defect accumulation phase of the simulation is not even finished. 

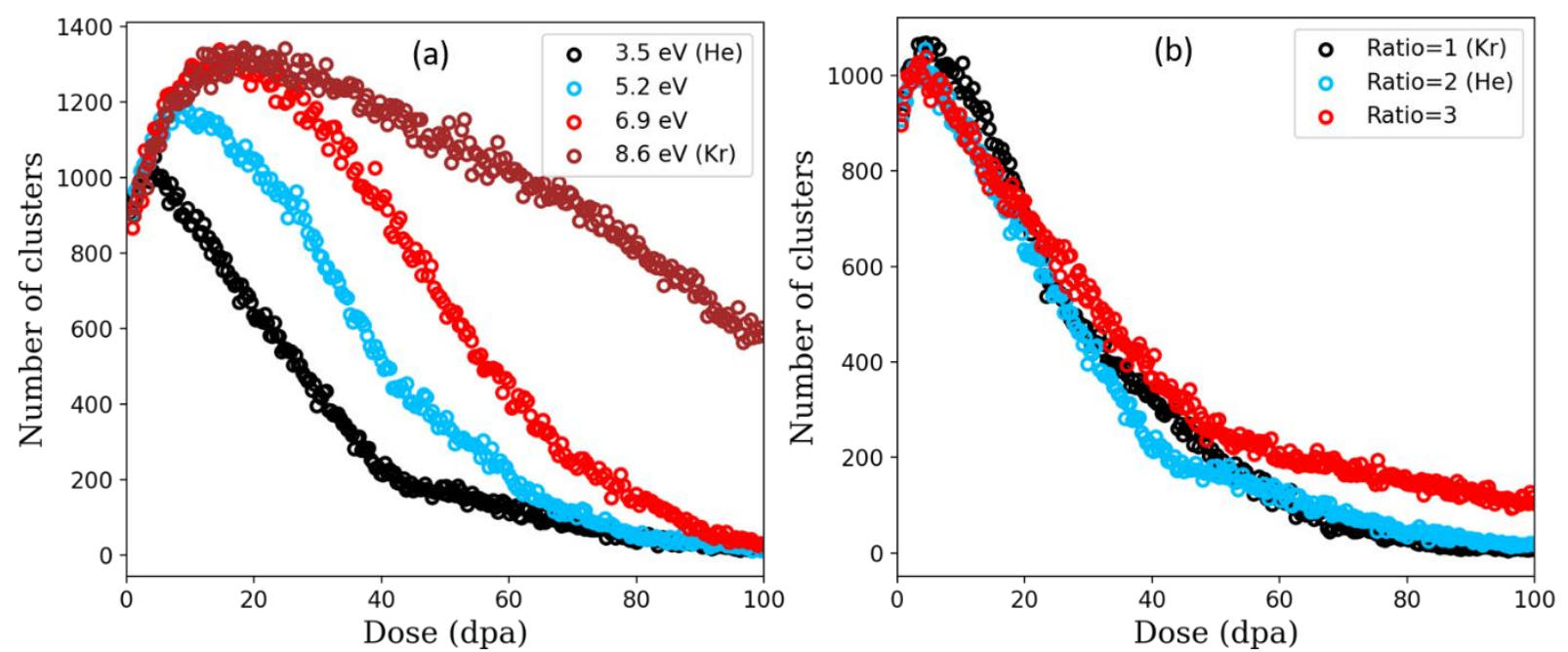

Figure S5. Number of vacancy clusters as a function of irradiation dose, for various gas-(a) vacancy binding energies (b) recombination limit ratio.

\section{The measurement of gas bubble constant, bubble size and distribution by synchrotron SAXS}

More descriptions of the models used to fit the SAXS patterns and extract particle size and distribution are provided here. The peak shown in Figure 3 was fitted with a gaussian distribution to determine the location and Full Width at Half Maximum. The lattice parameter was then determined by $a_{\text {bubble }}=i_{\text {bubble }} \times \sqrt{h^{2}+k^{2}+l^{2}}$ (with (hkl) (110)). Here, a $a_{\text {bubble }}$ is lattice constant and $i_{\text {bubble }}$ is the inter-bubble distance.

In SAXS, the scattering of the incident X-ray beam is the result of interactions with target electrons and variations in the scattered intensity resulting from the electron density fluctuations between the scattering objects and the background or host matrix. For a polydisperse spherical scattering system, with a size distribution $\mathrm{f}(\mathrm{r})$ and electron density $\rho$, embedded in a medium with electron density $\rho_{0}$, the scattering intensity $\mathrm{I}(\mathrm{Q})$ is given by:

$$
\mathrm{I}(\mathrm{q})=\mathrm{V}^{2} \times|\Delta \rho|^{2} \mathrm{~F}(\mathrm{q}) \times \mathrm{S}(\mathrm{q})
$$

Here is $\mathrm{V}$ the volume of the scattering particles, $(\Delta \rho)^{2}$ is the square of the difference in electron density between the particle and the background media $\left(\Delta \rho=\rho-\rho_{0}\right)$ and $\mathrm{F}(\mathrm{q})$ is the form/shape factor.

\section{References}

(1) Sun, C.; Sprouster, D. J.; Zhang, Y.; Chen, D.; Wang, Y.; Ecker, L. E.; Gan, J. Formation Window of Gas Bubble Superlattice in Molybdenum under Ion Implantation. Physical Review Materials 2019, 3 (10), 103607.

(2) Deo, C. S.; Okuniewski, M. A.; Srivilliputhur, S. G.; Maloy, S. A.; Baskes, M. I.; James, M. R.; Stubbins, J. F. Helium Bubble Nucleation in Bcc Iron Studied by Kinetic Monte Carlo Simulations. Journal of Nuclear Materials 2007, 361 (2-3 SPEC. ISS.), 141-148.

(3) Gao, Y.; Zhang, Y.; Schwen, D.; Jiang, C.; Sun, C.; Gan, J.; Bai, X.-M. Theoretical Prediction and Atomic Kinetic Monte Carlo Simulations of Void Superlattice SelfOrganization under Irradiation. Scientific Reports 2018, 8 (1), 6629. 
(4) Runevall, O.; Sandberg, N. Helium Cluster Dissolution in Molybdenum. Journal of Physics Condensed Matter 2009, 21 (33).

(5) Zhang, P.; Zou, T.; Zhao, J. He-He and He-Metal Interactions in Transition Metals from First-Principles. Journal of Nuclear Materials 2015, 467, 465-471.

(6) Jiang, C.; Zhang, Y.; Gao, Y.; Gan, J. Ab Initio Theory of Noble Gas Atoms in Bcc Transition Metals. Physical Chemistry Chemical Physics 2018, 20 (25), 17048-17058. 